\title{
Boundary effects in Cherenkov radiation
}

\author{
F. J. García de Abajo, ${ }^{1, *}$ A. Rivacoba, ${ }^{1,2}$ N. Zabala, ${ }^{1,3}$ and N. Yamamoto ${ }^{4}$ \\ ${ }^{1}$ Centro Mixto CSIC-UPV/EHU and Donostia International Physics Center (DIPC), Apartado Postal 1072, 20080 San Sebastián, Spain \\ ${ }^{2}$ Departamento de Física de Materiales, Facultad de Química, Universidad del País Vasco, Apartado Postal 1072, \\ 20080 San Sebastián, Spain \\ ${ }^{3}$ Departamento de Electricidad y Electrónica, Facultad de Ciencias, Universidad del País Vasco, Apartado Postal 644, \\ 48080 Bilbao, Spain \\ ${ }^{4}$ Physics Department, Tokyo Institute of Technology, Oh-okayama, Meguro-ku, Tokyo 152, Japan \\ (Received 27 February 2003; revised manuscript received 6 November 2003; published 19 April 2004)
}

\begin{abstract}
The effect of dielectric boundaries on the Cherenkov radiation (CR) produced when a fast point charge moves inside or near a material is analyzed for different shapes of the sample. Calculations are offered for a charge moving near both planar and nonplanar surfaces. CR is found to be produced even when the external charge moves outside a semi-infinite medium. For charges moving near planar boundaries, the reflected radiation interferes with the direct CR, leading to oscillations in the emission probability as a function of the impact parameter relative to the interfaces. Thin-film guided modes are excited by penetrating electrons and our calculations agree reasonably well with available experiments. The bulk limit in the emission probability is recovered for charges passing by the center of cylinders or spheres of increasingly large radius. Recent experiments of energy loss of electrons passing near dielectric spheres are explained thanks to the inclusion of retardation effects in the sphere response. These effects lead to an efficient channel of radiative energy losses. Finally, the diffraction of $\mathrm{CR}$ in void inclusions is proposed as a tool for providing information on otherwise inaccessible buried structures.
\end{abstract}

DOI: 10.1103/PhysRevB.69.155420

PACS number(s): 41.60.Bq, 41.20.-q, 41.75.Ht

\section{INTRODUCTION}

The electromagnetic field accompanying a charge freely moving in vacuum is entirely evanescent. However, if the charge moves inside a transparent material with constant velocity $v$ larger than the speed of light in the medium, coupling to propagating modes becomes possible and radiation is produced along the directions of the so-called Cherenkov cone. That condition can be restated as

$$
v>c / \sqrt{\epsilon},
$$

where $\epsilon$ is the dielectric function of the material under consideration. When Eq. (1) is fulfilled, the number of photons emitted by a unit charge per unit of path length is given by the well-known formula ${ }^{1}$

$$
P_{1}^{\text {bulk }}=\frac{1}{c^{2}}-\frac{1}{v^{2} \epsilon},
$$

which depends upon the photon wavelength exclusively via $\epsilon$.

This effect was discovered in 1934 by Pavel Cherenkov ${ }^{2}$ and its theoretical interpretation dates back to $1937 .^{3}$ The theory was later generalized to account for dispersive media ${ }^{4}$ and more sophisticated descriptions have been produced since then. ${ }^{5,6}$

The Cherenkov effect is extensively used to detect charged-particle byproducts resulting from energetic subatomic collisions in particle detectors. ${ }^{7-10}$ Also, this type of radiation has been observed in more exotic systems, such as Josephson junctions. ${ }^{11}$ In addition to the Cherenkov (wave) cone, a group cone of much smaller aperture has been predicted to occur in strongly dispersive active media. ${ }^{12}$
Among some exotic effects associated to this type of radiation, one can mention that the interaction of an external source of radiation with Cherenkov light can give rise to resonant scattering of the latter. ${ }^{13}$ Also, external radiation can be used to accelerate charged particles in what has been named inverse Cherenkov effect. ${ }^{14}$

The condition (1) arises from matching the allowed values of energy and momentum transfer between the external charge and the medium. However, Cherenkov radiation (CR) has been recently observed at subluminal velocities as well, thanks to the extra momentum supplied by phonons to make the energy-momentum matching possible. ${ }^{15}$

CR can be particularly important for fast electrons such as those used in transmission electron microscopes. Indeed, there are many technologically interesting materials that satisfy the Cherenkov condition (1) at accelerating voltages of $200 \mathrm{kV}$ (e.g., silicon and silica).

The purpose of this work is to analyze the effect of dielectric boundaries on the CR. In the presence of finite objects, the emission probability is no longer given by Eq. (2), and in particular, it will be shown to exhibit an explicit dependence on the wavelength via reflections at the boundaries. Previous studies on the subject include charges moving parallel to a surface ${ }^{16}$ or crossing a dielectric slab. ${ }^{17,18}$ More complicated systems have also been investigated theoretically, such as the electron bunches moving in waveguides, ${ }^{19}$ the CR produced by solitons in optical fibers, ${ }^{20,21} \mathrm{CR}$ from layered structures, ${ }^{22,23} \mathrm{CR}$ in photonic crystals, ${ }^{24,25}$ and the related phenomenon of Smith-Purcell radiation in strings of spheres $^{26}$ and photonic crystals. ${ }^{27,28}$ Finite-trajectory effects can be conveniently described by simple expressions derived from Tamm's theory ${ }^{4}$ in the short wavelength limit when most of the trajectory runs many wavelengths away from the boundaries. This has been applied to explain several experi- 
ments of practical interest in ultrarelativistic ion-beam characterization, where new phenomena related to finitetrajectory effects have been observed near the threshold condition (1). ${ }^{9}$ However, we shall be concerned with impact parameters and sample dimensions comparable to the wavelength in the present work, so that our theory retains all the complications of Maxwell's equations in the near- and intermediate-field regimes.

The charges under consideration will be assumed to be swift (e.g., relativistic ions and electrons), so that the eventual modification in their trajectories as a result of interaction with the samples will be neglected. In particular, image forces (these originate from reflected field contributions to the total induced field) can produce bending of the projectile trajectories, but we will overlook this effect, which should be small for swifting enough particles, as we are interested in the loss spectra and light emission distributions, and not so much in the angular distribution of the outgoing charges.

We will analyze the $\mathrm{CR}$ produced by fast charges moving near or inside a material bound within one particular spatial direction by planar surfaces (Sec. II), and also dielectrics bound in two or three spatial directions by cylindrical, spherical, and more complicated surfaces (Sec. III). Gaussian atomic units (i.e., $\hbar=m=e=1$ ) will be used throughout the paper unless otherwise specified. Nonmagnetic materials $(\mu=1)$ will be considered from now on.

\section{DIELECTRICS BOUND IN ONE SPATIAL DIRECTION: PLANAR BOUNDARIES}

When a charge moves parallel to a planar surface inside a dielectric under condition (1), the CR is reflected on the inner boundary and this produces interference effects that can be observed in the energy loss of the charge. If nonabsorbing, transparent materials are considered, the photon emission probability has to be equal to the energy loss probability, since photon emission is the only mechanism that can produce energy loss. Therefore, we will calculate the emission probability from the energy loss, which arises in turn from the retarding force produced by the induced electric field acting back on the charge. The induced field will be expressed in terms of reflection coefficients in the case of planar boundaries. This will be done following analytical methods that can find a broader field of applications.

To be more specific, let us first consider a unit point charge moving inside a homogeneous medium $j$, characterized by its dielectric function $\boldsymbol{\epsilon}_{j}$, with velocity $v$ along the $x$ direction, parallel to one or more planar interfaces. The interfaces will be chosen to be normal with respect to the $z$ axis and the charge trajectory will be described by $\mathbf{r}_{t}$ $=\left(v t, 0, z_{0}\right)$. Then, the electric field in the medium can be decomposed into a bulk direct term $\mathbf{E}_{j}^{\text {bulk }}$ (i.e., the field that would be produced if the charge were moving inside the bulk of an infinite medium $j$ ) plus the field resulting from the reflection at the boundaries $\mathbf{E}^{\text {boundary }}$, that is,
The direct field admits an analytical decomposition in terms of $s$ and $p$ waves, which takes the form, in frequency space $\omega$,

$$
\mathbf{E}_{j}^{\text {bulk }}(\mathbf{r})=\int d Q_{y} \sum_{\sigma} e^{\mathrm{i} \mathbf{K}_{\mathbf{Q}}^{ \pm} \cdot \mathbf{r}} E_{\mathbf{Q}, \sigma}^{j \pm} \hat{\mathbf{e}}_{\mathbf{Q}, \sigma}^{j \pm}
$$

The momentum of one of such plane waves,

$$
\mathbf{K}_{\mathbf{Q}}^{ \pm}=\left(\mathbf{Q}, \pm k_{z j}\right),
$$

forms an orthonormal basis set together with the normalized polarization vectors

$$
\hat{\mathbf{e}}_{\mathbf{Q}, s}^{j \pm}=\frac{1}{Q}\left(-Q_{y}, Q_{x}, 0\right)
$$

and

$$
\hat{\mathbf{e}}_{\mathbf{Q}, p}^{j \pm}=\frac{1}{k_{j} Q}\left( \pm k_{z j} \mathbf{Q},-Q^{2}\right) .
$$

Here, $k_{z j}=\sqrt{k_{j}^{2}-Q^{2}}, k_{j}=(\omega / c) \sqrt{\epsilon_{j}}$ is the momentum of the light in medium $j$, and $Q_{x}=\omega / v$. Since $\operatorname{Im}\left\{\epsilon_{j}\right\}>0$ (even if it is infinitesimal), the above square root can be chosen such that both the real and the imaginary parts of $k_{z j}$ are simultaneously positive, so that the upper (lower) sign must be adopted for positive (negative) values of $z-z_{0}$ in order to guarantee that the fields vanish in the $|z| \rightarrow \infty$ limit. The coefficients of this plane wave expansion $\operatorname{are}^{28}$

$$
E_{\mathbf{Q}, s}^{j \pm}=\frac{Q_{y} \omega}{Q k_{z j} c^{2}} e^{\mp i k_{z j} z_{0}}
$$

and

$$
E_{\mathbf{Q}, p}^{j \pm}=\mp \frac{k_{j}}{v Q \epsilon_{j}} e^{\mp \mathrm{i} k_{z j} z_{0}}
$$

\section{A. Semi-infinite media}

Applying the above formalism to the case of a charge moving above a single interface located at $z=0$ (i.e., the impact parameter $z_{0}$ is positive), the reflected field can be easily obtained from the direct field by using the reflection coefficients of the planar interface (as seen from inside medium $j$, which occupies the $z>0$ region where the charge is moving), ${ }^{1}$

$$
R_{j j^{\prime}}^{s}=\left(k_{z j}-k_{z j^{\prime}}\right) /\left(k_{z j}+k_{z j^{\prime}}\right)
$$

and

$$
R_{j j^{\prime}}^{p}=\left(\epsilon_{j^{\prime}} k_{z j}-\epsilon_{j} k_{z j^{\prime}}\right) /\left(\epsilon_{j^{\prime}} k_{z j}+\epsilon_{j} k_{z j^{\prime}}\right),
$$

where $j^{\prime}$ is the medium on the $z<0$ side of the interface. One finds

$$
\mathbf{E}^{\text {boundary }}(\mathbf{r})=\int d Q_{y} \sum_{\sigma} e^{i \mathbf{K}_{\mathbf{Q}}^{+} \cdot \mathbf{r}} R_{j j^{\prime}}^{\sigma} E_{\mathbf{Q}, \sigma}^{j-} \hat{\mathbf{e}}_{\mathbf{Q}, \sigma}^{j+},
$$


where use has been made of the fact that the direct field is traveling downwards, whereas the reflected field propagates in the direction of increasing $z$ 's, back to the external charge.

The energy loss probability is then obtained from the force produced by the induced field on the external charge. The energy loss per unit of path length can then be written as

$$
\frac{-1}{L} \int d t \mathbf{v} \cdot \mathbf{E}^{\text {ind }}\left(\mathbf{r}_{t}, t\right)=\int_{0}^{\infty} \omega d \omega P(\omega)
$$

where

$$
P(\omega)=\frac{-1}{\pi \omega L} \int d t \operatorname{Re}\left[e^{-\mathrm{i} \omega t} \mathbf{v} \cdot \mathbf{E}^{\mathrm{ind}}\left(\mathbf{r}_{t}, \omega\right)\right]
$$

is the loss probability and $L$ is the length of the trajectory. Following Ref. 29, this quantity can be in turn decomposed into contributions coming both from the bulk direct field $\mathbf{E}_{j}^{\text {bulk }}$ and from the field reflected at the boundaries $\mathbf{E}^{\text {boundary }}$ :

$$
P=P^{\text {bulk }}+P^{\text {boundary }} .
$$

The former can be worked out from Eq. (3) to recover Eq. (2) if medium $j$ is transparent (with $\epsilon_{j}$ substituted for $\epsilon$ ), provided the Cherenkov condition (1) is fulfilled. ${ }^{1,29}$ The remaining contribution to Eq. (10) results from inserting Eq. (8) into Eq. (9). One obtains

$$
\begin{aligned}
P^{\text {boundary }}(\omega)= & \frac{2}{\pi v^{2}} \int_{0}^{\infty} \frac{d Q_{y}}{Q^{2}} \\
& \times \operatorname{Re}\left\{k_{z j} e^{2 i k_{z j} z_{0}}\left[\left(\frac{Q_{y} v}{k_{z j} c}\right)^{2} R_{j j^{\prime}}^{s}-\frac{1}{\epsilon_{j}} R_{j j^{\prime}}^{p}\right]\right\} .
\end{aligned}
$$

This expression, which is valid for arbitrary complex dielectric functions $\epsilon_{j}$ and $\epsilon_{j^{\prime}}$, has been obtained in a different way by García-Molina et al. ${ }^{30}$ although the derivation presented here is more straightforward and can be easily generalized to include more interfaces (see below).

Equation (11) has been used to obtain the loss probability for a point charge moving parallel to the interface of a material described by $\epsilon=3$ (e.g., alumina in the visible and near infrared) with vacuum, as shown in Fig. 1. The results have been normalized to the bulk limit [Eq. (2)]. Interestingly, CR is produced even when the charge is moving outside the material $\left(z_{0}>0\right)$, although the loss probability decays exponentially with increasing $z_{0}$, reflecting the evanescent nature of the field produced by the external charge in vacuum. ${ }^{16}$ However, when the charge moves inside the material, the direct field is radiative and it can be reflected at the inner surface and propagated back to the charge, giving rise to an interference pattern that extends well beyond the radiation wavelength $\lambda$. It should be noticed that the spatial frequency of these oscillations increases with increasing velocity [i.e., with increasing $\left.Q_{\perp}=(\omega / c) \sqrt{\epsilon-c^{2} / v^{2}}\right]$ as a result of the change in the orientation of the Cherenkov cone relative to the dielectric boundary (actually, $Q_{\perp}$ is the projection of the momentum of Cherenkov light along the surface normal). Near the surface, the contribution of evanescent waves to Eq.

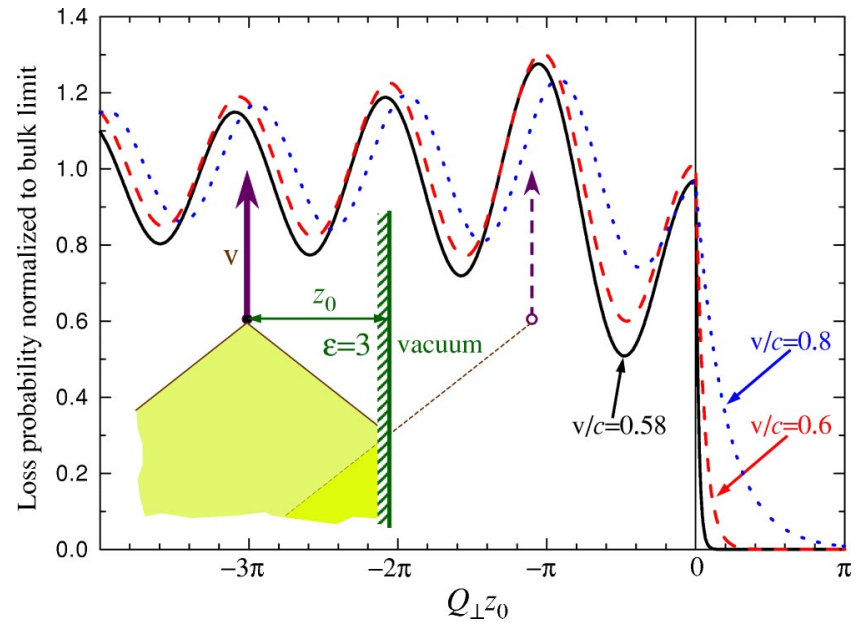

FIG. 1. (Color online) Cherenkov radiation (CR) emission probability near a planar interface. The charge is moving inside (outside) a material described by $\epsilon=3$ for $z_{0}<0\left(z_{0}>0\right)$ along a direction parallel to the surface. Different charge velocities have been considered, as shown by labels. The probability has been normalized to the bulk limit [Eq. (2)], while the impact parameter $z_{0}$ is normalized using the momentum of the light perpendicular to the trajectory, $Q_{\perp}=(\omega / c) \sqrt{\epsilon-c^{2} / v^{2}}$. The inset depicts the Cherenkov cone and its reflection at the inner boundary when the particle moves inside the medium. Total internal reflection of the CR at the surface confines the emission inside the material.

(11) can be substantial (i.e., for $Q_{y}>Q_{\perp}$ ), but deep inside the bulk the loss is dominated by the geometrical construction of the propagating Cherenkov cone and its reflection at the surface, as shown in the inset of Fig. 1. Therefore, the oscillations observed in the figure die off slowly like a Bessel function, a behavior similar to that predicted for two unit point charges ( $N$ charges in general) following laterally displaced trajectories in a homogeneous infinite medium, in which case the loss probability becomes, from a straightforward solution of Maxwell's equations,

$$
P_{N}^{\text {bulk }}=P_{1}^{\text {bulk }} \sum_{i i^{\prime}} \cos \left[\frac{\omega\left(r_{i}^{\|}-r_{i^{\prime}}^{\|}\right)}{v}\right] J_{0}\left[Q_{\perp}\left|\mathbf{r}_{i}^{\perp}-\mathbf{r}_{i^{\prime}}^{\perp}\right|\right],
$$

where the double sum is extended over the moving particles, and $r^{\|}$and $\mathbf{r}^{\perp}$ denote coordinates parallel and perpendicular to the velocity vector, respectively.

\section{B. Slabs: parallel trajectories}

The case of a thin film can be also analyzed within the above formalism by considering the multiple successive reflections of plane-wave components at both inner boundaries. We will consider a unit point charge moving inside a film of thickness $d$ described by $\epsilon$ (medium $j=1$ ) and surrounded by vacuum (medium $j^{\prime}=0$ ), with the trajectory directed parallel to the surfaces at a distance $z_{0}$ from the center of the film. Successive reflections can be summed analytically in this Fabry-Perot-like model to yield the following electric field inside the film: 


$$
\begin{aligned}
\mathbf{E}(\mathbf{r})= & \int d Q_{y} \sum_{\sigma} \frac{1}{1-\left(R_{21}^{\sigma} e^{\mathrm{i} k_{z 1} d}\right)^{2}} \\
& \times\left\{e^{\mathrm{i} \mathbf{K}_{\mathbf{Q}}^{+} \cdot \mathbf{r}} \hat{\mathbf{e}}_{\mathbf{Q}, \sigma}^{2+}\left[E_{\mathbf{Q}, \sigma}^{2+}+R_{21}^{\sigma} E_{\mathbf{Q}, \sigma}^{2-} e^{\mathrm{i} k_{z 1}\left(d+z_{0}\right)}\right]\right. \\
& \left.+e^{\mathrm{i} \mathbf{K}_{\mathbf{Q}}^{-} \cdot \mathbf{r}} \hat{\mathbf{e}}_{\mathbf{Q}, \sigma}^{2-}\left[E_{\mathbf{Q}, \sigma}^{2-}+R_{21}^{\sigma} E_{\mathbf{Q}, \sigma}^{2+} e^{i k_{z 1}\left(d-z_{0}\right)}\right]\right\},
\end{aligned}
$$

where $k_{z 1}=\sqrt{\epsilon \omega^{2} / c^{2}-Q^{2}}, k_{z 0}=\sqrt{\omega^{2} / c^{2}-Q^{2}}$, and $R_{21}^{s}$ and $R_{21}^{p}$ are given by Eqs. (6) and (7), respectively. The loss probability obtained by inserting Eq. (12) into Eq. (9) becomes, after discounting the bulk contribution $P^{\text {bulk }}$,

$$
\begin{aligned}
P^{\text {boundary }}(\omega)= & \frac{4}{\pi v^{2}} \int_{0}^{\infty} \frac{d Q_{y}}{Q^{2}} \operatorname{Re}\left\{\frac{1}{k_{z 1}}\left(\frac{Q_{y} v}{c}\right)^{2}\right. \\
& \times\left[\frac{R_{21}^{s} e^{\mathrm{i} k_{z 1} d} \cos \left(2 k_{z 1} z_{0}\right)+1}{1-\left(R_{21}^{s} e^{\mathrm{i} k_{z 1} d}\right)^{2}}-1\right] \\
& \left.-\frac{k_{z 1}}{\epsilon}\left[\frac{R_{21}^{p} e^{\mathrm{i} k_{z 1} d} \cos \left(2 k_{z 1} z_{0}\right)-1}{1-\left(R_{21}^{p} e^{\mathrm{i} k_{z 1} d}\right)^{2}}+1\right]\right\},
\end{aligned}
$$

Note that $Q \geqslant \omega / v>\omega / c$, so that $k_{z 0}$ is pure imaginary. Furthermore, if $\epsilon$ is real, $k_{z 1}$ is pure imaginary as well for $Q_{y}$ $>Q_{\perp}$ (this corresponds to evanescent and amplifying wave components inside the film) and consequently $R_{21}^{\sigma}$ is real. In that case, the expression inside the curly brackets of Eq. (13) is pure imaginary and the integrand vanishes. Therefore, we are only left with the $Q_{y}<Q_{\perp}$ part of the integral, where $k_{z 1}$ is real, $\left|R_{21}^{\sigma}\right|=1$, and one finds

$$
P_{\text {nonres. }}^{\text {boundary }}=\frac{-2}{\pi v^{2}} \int_{0}^{Q_{\perp}} \frac{d Q_{y}}{Q^{2}}\left[\frac{k_{z 1}}{\epsilon}+\frac{1}{k_{z 1}}\left(\frac{Q_{y} v}{c}\right)^{2}\right]=-P_{1}^{\text {bulk }}
$$

(the label nonres. stands for nonresonant; see below) which cancels out the bulk contribution altogether independently of the values of $d$ and $z_{0}$. The same result can be obtained from previous formulations of this problem, ${ }^{23,31}$ and similar conclusions are also drawn for parallel external trajectories.

Actually, $P_{\text {nonres. }}^{\text {boundary }}$ is only a part of $P^{\text {boundary }}$. There is another term originating in the zeros of the denominators of Eq. (13), which give rise to $\delta$ functions that provide the only nonvanishing contribution to the loss probability. This is a resonant contribution, which results from the coupling between the fast charge and the guided modes of the film, whose dispersion relations are precisely given by the zeros just noted, $R_{21}^{\sigma} e^{\mathrm{i} k_{z 1} d}= \pm 1$. These relations define a discrete set of $\omega(Q)$ curves, which are represented by crosses $(\sigma$ $=p)$ and dots $(\sigma=s)$ in the left part of Fig. 2 for $\epsilon=11.9$ (this represents silicon at a photon wavelength $\lambda$ $=1.55 \mu \mathrm{m}$, chosen for its relevance to optical communications technology and also as a representative case of a highdielectric-constant material).

It is worth mentioning that the Cherenkov condition (1) for inner trajectories parallel to the surface implies that the condition of total internal reflection is satisfied for CR. Thus,

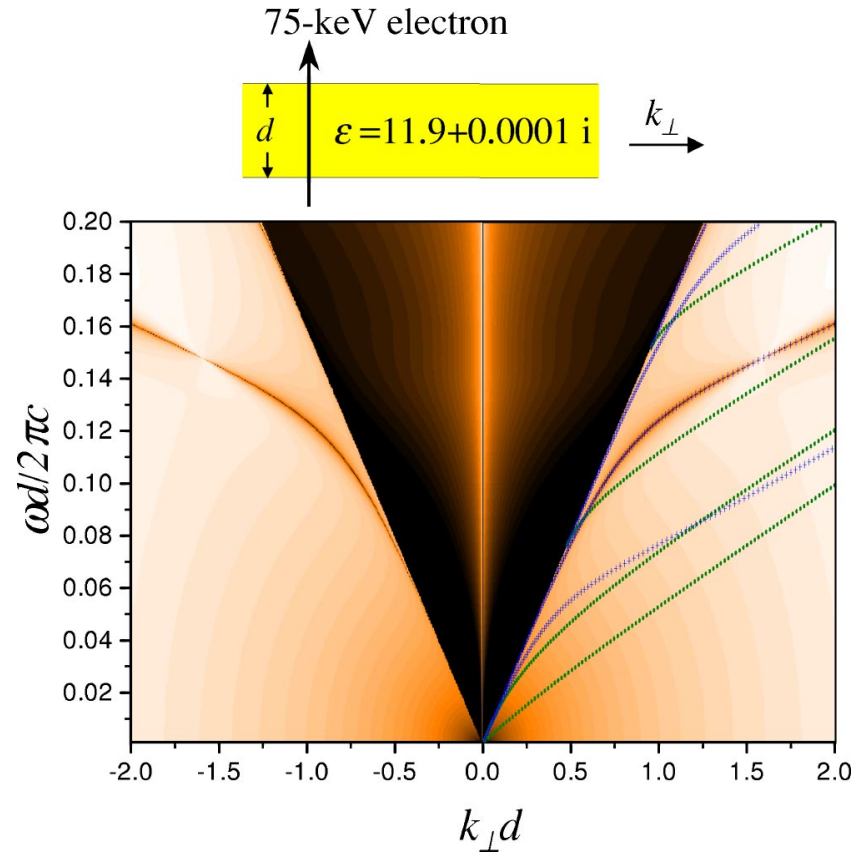

FIG. 2. (Color online) Electron energy loss probability as a function of energy and momentum transfer for $75 \mathrm{keV}$ electrons traversing a film of thickness $d$ characterized by a dielectric function $\epsilon=11.9+0.0001$ i. Darker regions correspond to higher probability. The electron beam is directed along the film normal and the momentum transfer $k_{\perp}$ is perpendicular to the trajectory. The guided modes of the film are represented in the right half part of the figure: crosses for $p$-polarized modes ( $H$ parallel to the film) and dots for $s$-polarized modes ( $E$ parallel to the film). Only even-order $p$ modes are excited by the electrons.

$\mathrm{CR}$ is confined within the film, and destructive interference between successive reflections at the film surfaces leads to lossless propagation of the charge except for specific energy and momentum transfers that match the mentioned dispersion relation for guided modes.

\section{Slabs: penetrating trajectories}

At this point, a distinction has to be made between CR and transition radiation (TR). Whereas the former is clearly connected to coupling of the external charge to propagating modes in an infinite system, the latter occurs whenever the charge crosses a physical interface. ${ }^{32}$ This distinction becomes fuzzy when the charge interacts with objects of finite size along the direction of propagation. The effect of termination of a medium gives rise to TR, but CR generated away from the interface can be partially transmitted outside the material. This will be discussed in Sec. III for cylinders and spheres.

Let us examine the case of unit charges transmitted through thin films. Detailed expressions for the photonemission probability have been given in the past for charges crossing thin films. ${ }^{33}$ For completeness, we will derive related expressions within the present formalism and apply them to calculate energy loss probabilities, including multilayers of lossy and anisotropic materials, and comparison with actual electron-energy-loss spectroscopy(EELS) experi- 
ments in thin films made of this kind of materials will be offered. The multilayer geometry has been studied in the past by means of a complicated computer algebra scheme, ${ }^{23}$ but we will use a more straight-forward formulation that leads to simple expressions that will be used to explain available experimental results. ${ }^{34,35}$ To study penetrating trajectories one can describe the field inside each homogeneous region of space by the corresponding $\mathbf{E}_{j}^{\text {bulk }}$ field for the infinite medium plus plane waves that are used to match the boundary conditions at the interfaces. For a slab of material $j=1$ contained in the $0<z<d$ region and for a trajectory defined by $\mathbf{r}_{t}=(0,0, v t)$, the electric field can be written in frequency space $\omega$ as

$$
\mathbf{E}(\mathbf{r})=\int \frac{d^{2} \mathbf{Q}}{(2 \pi)^{2}} \mathbf{E}(\mathbf{Q}, z) e^{\mathrm{i} \mathbf{Q} \cdot(x, y)},
$$

where

$$
\begin{aligned}
\mathbf{E}(\mathbf{Q}, z) & =\mathbf{E}_{0}^{\text {bulk }}(\mathbf{Q}, z)+A e^{-\mathrm{i} k_{z 0} z} \hat{\mathbf{e}}_{\mathbf{Q}, p}^{0-} \quad z<0, \\
& =\mathbf{E}_{1}^{\text {bulk }}(\mathbf{Q}, z)+\sum_{\alpha= \pm} C_{\alpha} e^{\alpha i k_{z 1} z} \hat{\mathbf{e}}_{\mathbf{Q}, p}^{1 \alpha} \quad 0<z<d, \\
& =\mathbf{E}_{0}^{\text {bulk }}(\mathbf{Q}, z)+B e^{\mathrm{i} k_{z 0} z} \hat{\mathbf{e}}_{\mathbf{Q}, p}^{0+}, \quad z>d .
\end{aligned}
$$

Here,

$$
\mathbf{E}_{j}^{\text {bulk }}(\mathbf{Q}, z)=\frac{4 \pi \mathrm{i} e^{\mathrm{i} \omega z / v}}{v \epsilon_{j}\left(k_{j}^{2}-q^{2}\right)}\left(\mathbf{q}-\mathbf{v} k_{j}^{2} / \omega\right)
$$

is the bulk field, $j=0$ refers to the vacuum on either side of the film $\left(\epsilon_{0}=1\right)$, and $\mathbf{q}=(\mathbf{Q}, \omega / v)$. The signs in the exponents of the terms proportional to $A$ and $B$ are chosen such that they represent induced waves that propagate away from the film. Note that only $p$ polarized waves are involved in the above expression [see Eq. (5)], since $s$ waves are perpendicular to the bulk electric field: $\hat{\mathbf{e}}_{\mathbf{Q}, s}^{j \pm} \cdot \mathbf{E}_{j}^{\text {bulk }}(\mathbf{Q}, z)=0$.

The boundary conditions for the electromagnetic field reduce to the continuity of the parallel components of the electric and magnetic field at the film surfaces $z=0$ and $z=d$. This implies automatically the continuity of the normal displacement and the normal magnetic field. Moreover, our electric field has $p$ character, so that the magnetic field obtained from the above expressions by using Faraday's law has $s$ character, and in particular

$$
\mathbf{H}_{j}^{\text {bulk }}(\mathbf{Q}, z)=\frac{(4 \pi \mathrm{i} Q / c) e^{\mathrm{i} \omega z / v}}{v \epsilon_{j}\left(k_{j}^{2}-q^{2}\right)} \hat{\mathbf{e}}_{\mathbf{Q}, s}^{j+} .
$$

Therefore, the parallel components of the electric field are all directed along $\mathbf{Q}$ and those of the magnetic field are directed perpendicular to $\mathbf{Q}$. So, we have two conditions for the continuity of the nonvanishing parallel component of the electric field (one at each film surface), and two more conditions for the magnetic field, totalling four linear equations that involve the four unknown coefficients $A, B$, and $C_{ \pm}$. This system is solved analytically to yield these coefficients. The procedure is straightforward, but we omit the details, since the resulting expressions are cumbersome and do not add much to the physics.

Inserting Eq. (15) into Eq. (9), one can separate the loss probability into contributions coming from different momentum transfers $\mathbf{Q}$ parallel to the film surfaces according to

$$
P(\omega)=\int \frac{d^{2} \mathbf{Q}}{(2 \pi)^{2}} P(\mathbf{Q}, \omega) .
$$

Finally, the integration over the time in Eq. (9) can be performed analytically to yield

$$
\begin{aligned}
P^{\text {boundary }}(\mathbf{Q}, \omega)= & \frac{-Q c}{\pi \omega^{2}} \operatorname{Im}\left\{\frac{A}{k_{z 0}+\omega / v}+\frac{B \mathrm{e}^{\mathrm{i}\left(k_{z 0}-\omega / v\right) d}}{k_{z 0}-\omega / v}\right. \\
& -\frac{C_{-}}{\sqrt{\epsilon_{1}}} \frac{\mathrm{e}^{-\mathrm{i}\left(k_{z 1}+\omega / v\right) d}-1}{k_{z 1}+\omega / v} \\
& \left.+\frac{C_{+}}{\sqrt{\epsilon_{1}}} \frac{\mathrm{e}^{\mathrm{i}\left(k_{z 1}-\omega / v\right) d}-1}{k_{z 1}-\omega / v}\right\}
\end{aligned}
$$

and $P^{\text {bulk }}(\mathbf{Q}, \omega)=P_{1}^{\text {bulk }} \operatorname{Im}\left\{4 d /\left(\omega^{2} / v^{2}-k_{z 1}^{2}\right)\right\}$. The latter takes nonzero values at the zeros of the denominator (i.e., $Q=Q_{\perp}$, with $Q_{\perp}$ as defined in Sec. II A), but this is compensated by some of the poles in $P^{\text {boundary }}$.

These analytical expressions have been used to obtain the contour plot of Fig. 2 for $75 \mathrm{keV}$ electrons transmitted through Si films $\left(\epsilon_{1}=\epsilon=11.9+0.0001 i\right)$, where one can observe the excitation of guided modes. The small imaginary part in $\epsilon_{1}$ has been added to broaden these guided modes just for representation purposes. The contour plot of Fig. 2 represents the intensity (darker regions correspond to higher intensity) as a function of the energy loss $\omega$ and perpendicular (with respect to the electron trajectory) momentum transfer $k_{\perp}$ (=Q for normal incidence).

The angle of emission of CR relative to the trajectory in bulk $\mathrm{Si}$ at $75 \mathrm{keV}\left(53.7^{\circ}\right)$ is larger than the angle of total internal reflection $\left(16.9^{\circ}\right)$, and therefore, no bulk CR is allowed to exit the film in Fig. 2. Instead, CR is confined inside the film, but only at discretized guided-mode frequencies that lie outside the light cone. The dispersion relation of these modes has been represented in the right side of the figure (crosses and dots), where it is clear that the second $p$-polarized mode (crosses; $H$ field parallel to the film surfaces) is excited by the incoming electron. Further inspection reveals that only even-order $p$ modes are excited, which correspond to odd-parity modes along the film normal direction. Equation (16) anticipated already that only coupling to $p$ modes is possible under normal incidence, and even-parity modes are excluded due to symmetry mismatch.

Since no CR exits the film, the light cone (central structure) must contain contributions originating in TR. The emission seems to vanish close to the film normal $\left(k_{\perp}=0\right)$, whereas it is enhanced at low take-off angles (i.e., close to the light cone boundary). This pattern suggests the inducement of a sudden dipole as the electron crosses each film surface, giving rise to dipolelike emission characteristic of TR. 


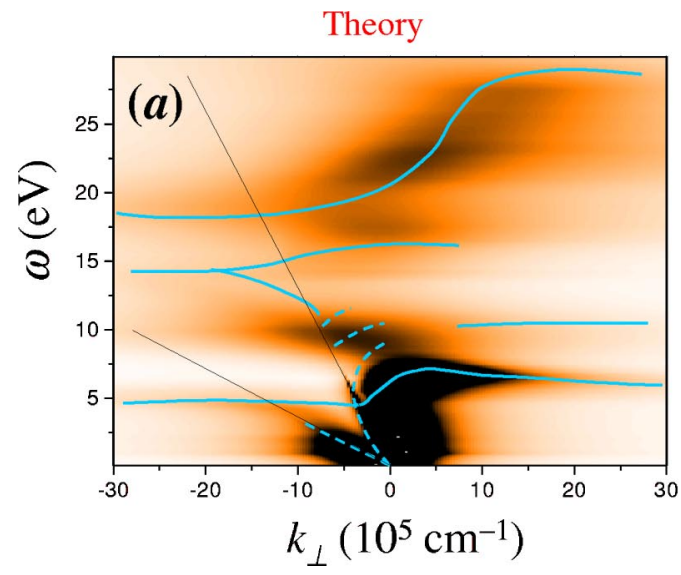

Experiment: Chen and Silcox, 1975

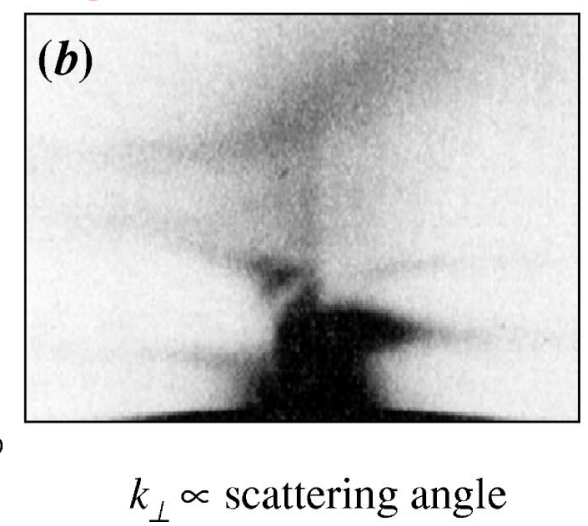

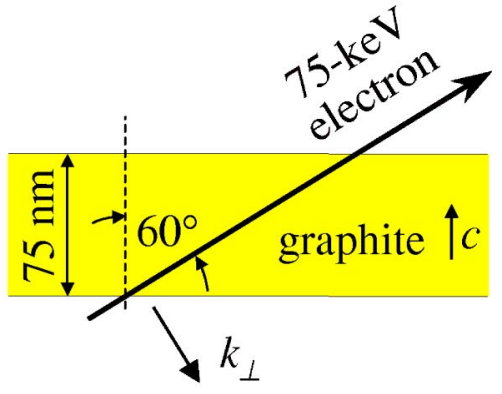

FIG. 3. (Color online) (a) Electron energy loss probability calculated as a function of energy and momentum transfer for $75 \mathrm{keV}$ electrons traversing a $75 \mathrm{~nm}$ graphite film with an angle of incidence of $60^{\circ}$ with respect to the film normal. Darker regions correspond to higher probability (in logarithmic scale). The momentum transfer $k_{\perp}$ is taken perpendicular to the electron velocity and it is contained in the plane defined by the film normal and the direction of incidence. (b) Experimental data for the same system as in (a), taken from Chen and Silcox, ${ }^{35}$ where $k_{\perp}$ is directly connected to the electron scattering angle. The boundaries of the light cone have been represented by solid straight lines in (a). Also, the intensity maxima observed in (b) are represented in (a) by solid and dashed curves, as given in Ref. 35 .

A direct proof of coupling to guided modes was provided by Chen and $\mathrm{Silcox}^{35}$ in an elegant experiment where they used graphite films under oblique incidence (a tilt angle of $\alpha=60^{\circ}$ ). Their results for the geometry sketched in the right hand side of Fig. 3 are reproduced in Fig. 3(b). Their measured intensity maxima are showed in Fig. 3(a) (solid and broken curves), where they are compared with our calculations. The bulk field $\mathbf{E}_{j}^{\text {bulk }}$ takes now a more complicated form as compared with the case of normal incidence, but the analytical calculation follows the same lines as explained above. Some details about the bulk field for oblique incidence in anisotropic materials are given in the Appendix. The dielectric function of graphite has been taken from frequency-dependent optical data. ${ }^{36}$ The observed guided modes are represented by broken curves, which are in reasonable agreement with the maxima obtained in the calculation, given the experimental uncertainty still remaining in the actual values of the dielectric function of graphite for polarization along its $c$ axis.

The loss intensity of Fig. 3 is given as a function of the momentum transfer $k_{\perp}$ perpendicular to the trajectory, which is in turn proportional to the electron scattering angle in the low-momentum limit under consideration. For a vector momentum transfer $\mathbf{q}=\left(k_{\perp}, 0, \omega / v\right)$ contained in the plane defined by the trajectory and the film normal, the component of the momentum parallel to the film surfaces is ${ }^{37} Q_{x}$ $=(\omega / v) \sin \alpha+k_{\perp} \cos \alpha$, and this is the relation actually used in plotting Fig. 3.

Radiation can also be produced in metallic films via coupling to low-momentum radiative modes ${ }^{38}$ as shown in Fig. 4 (again, optical data ${ }^{36}$ have been used to model the dielectric functions of the materials under consideration). This was first observed by Vincent and Silcox ${ }^{34}$ in aluminum thin films [see Figs. 4(c)-4(d)]. Our calculations [and Figs. 4(a) and 4(b)] show a general good agreement with the experiment, and in particular, they reproduce the position and dispersion relation of nonradiating surface plasmon modes $\omega^{ \pm}$and the mentioned radiative mode $\omega_{r}$ right above the bulk plasma frequency $\omega_{p}$. The details of the calculations are rather tedious but straightforward: we write down a system of equations similar to that of Eq. (16) for the continuity of the parallel components of the electric field and the magnetic field at each of the four interfaces in the film, with the bulk field specialized for oblique incidence as shown in Eqs. (A1) for $\epsilon_{j}^{\perp}=\epsilon_{j}^{\|}=\epsilon_{j}$; this leads to a system of linear equations in the expansion coefficients that is solved analytically; finally, the electric field is inserted into Eq. (9) and integrated along the trajectory to yield the angle and momentum resolved loss probability. The $2 \mathrm{~nm}$ thick oxide layers account for oxidation of aluminum and help improve the calculated results slightly with respect to the bare aluminum film, as compared with the experiment.

\section{DIELECTRICS BOUND IN TWO AND THREE SPATIAL DIRECTIONS: CYLINDRICAL AND SPHERICAL BOUNDARIES}

\section{A. Cylinders}

Let us first consider a quasistationary situation where a unit charge is moving with constant velocity $v$ along the axis of an infinite cylinder of radius $a$ and real dielectric function $\epsilon$ placed in vacuum. Using a formalism similar to the one employed in preceding section, with cylindrical waves rather than plane waves (only $m=0$ contributes due to the symmetry of the problem), the loss probability per unit of path length is found to be $\mathrm{b}^{39}$

$$
P=P_{1}^{\text {bulk }} \operatorname{Im}\left\{\frac{K_{0}(B) Y_{1}(A) \epsilon B+K_{1}(B) Y_{0}(A) A}{K_{0}(B) J_{1}(A) \epsilon B+K_{1}(B) J_{0}(A) A}\right\}
$$

where $P_{1}^{\text {bulk }}$ is given by Eq. (2), $A=(\omega a / v) \sqrt{\epsilon v^{2} / c^{2}-1}$, and $B=(\omega a / v) \sqrt{1-v^{2} / c^{2}}$. Under these conditions, all functions inside the Im operator take real values, so that the loss 

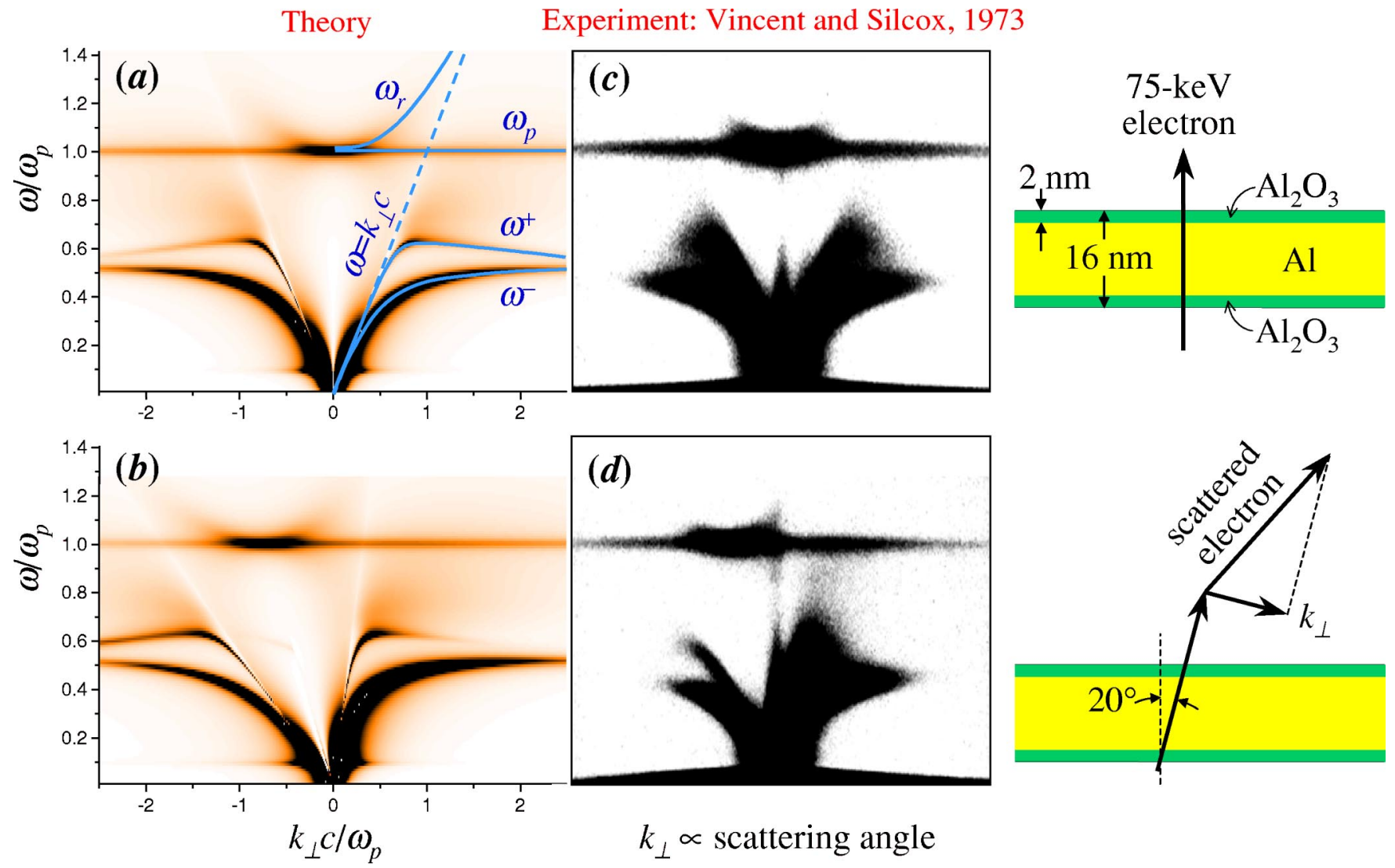

FIG. 4. (Color online) Same as Fig. 3 for a $16 \mathrm{~nm}$ aluminum film. (a) and (b) correspond to the present calculations, whereas (c) and (d) are experimental observations taken from Vincent and Silcox. ${ }^{34}$ The geometries under consideration are shown in the insets: incidence along the film normal in (a) and (c), and $20^{\circ}$ off-normal incidence in (b) and (d). The light line has been represented in (a) by a broken straight line, whereas some other characteristic features are indicated by solid curves: the nonradiative surface plasmon modes $\omega^{ \pm}$, the bulk plasmon $\omega_{p}=15 \mathrm{eV}$, and a radiative mode $\omega_{r}$ within the light cone. A $2 \mathrm{~nm}$ thick oxide layer is assumed on each side of the film.

probability vanishes altogether, unless the denominator becomes zero. Therefore, the confinement of the CR along the two spatial directions perpendicular to the trajectory results in discretization of the emission, which becomes possible only for wavelengths that resonate with the propagating modes of the cylinder (i.e., guided modes), confined in its interior by total internal reflection like in a wave guide. ${ }^{40}$ This situation is equivalent to the one encountered for trajectories parallel to a thin film [Eq. (13)]. Similar results are obtained for external trajectories parallel to a dielectric cylinder. $^{29}$

Next, we will consider both aloof and penetrating trajectories perpendicular to the cylinder. In an aloof geometry the external charge does not touch the material, and this configuration will give us further insight into the excitation of the mentioned guided modes, as shown below. For penetrating trajectories, we will recover the bulk limit of Eq. (2) as the diameter of the cylinders becomes arbitrarily large. However, although it is possible to do it, these situations are rather tedious to be explored by means of analytical methods.

Instead, we will use a numerical solution of Maxwell's equations based upon the versatile boundary element method (BEM), ${ }^{29,41}$ which consists in expressing the electromagnetic field in frequency space $\omega$ in terms of boundary charges and currents that are self-consistently obtained by applying the customary boundary conditions of the electromagnetic field in the presence of the external field set up by the moving charge. This method can be applied to complex geometries and the numerical effort that is involved is relatively small as compared to other methods of solution of Maxwell's equations because the problem is reduced to a set of surfaceintegral equations (the boundary conditions), which are essentially two dimensional. Furthermore, for translationallyinvariant interfaces (just as the cylinders considered in this section) one can work in momentum space $q$ with respect to the direction of translational invariance (say $z$ ), which reduces further the dimensionality of the problem to just onedimensional integral equations for each separate $q$ component. The integrals along the boundary contours are then discretized and the induced boundary sources are represented by a set of representative boundary points, leading to a set of linear equations that are solved by standard numerical techniques. As a result, the electric field within a certain medium $j$ takes the form ${ }^{29}$

$$
\begin{aligned}
\mathbf{E}(\mathbf{r})= & \mathbf{E}_{j}^{\mathrm{bulk}}(\mathbf{r})+\int \frac{d q}{\pi} \int d l\left[\frac{\mathrm{i} \omega}{c} \mathbf{h}_{j}(l, q)\right. \\
& \left.-\sigma_{j}(l, q) \nabla\right] K_{0}\left(\frac{q\left|\mathbf{R}-\mathbf{R}_{l}\right|}{\gamma_{j}}\right) e^{\mathrm{i} q z},
\end{aligned}
$$




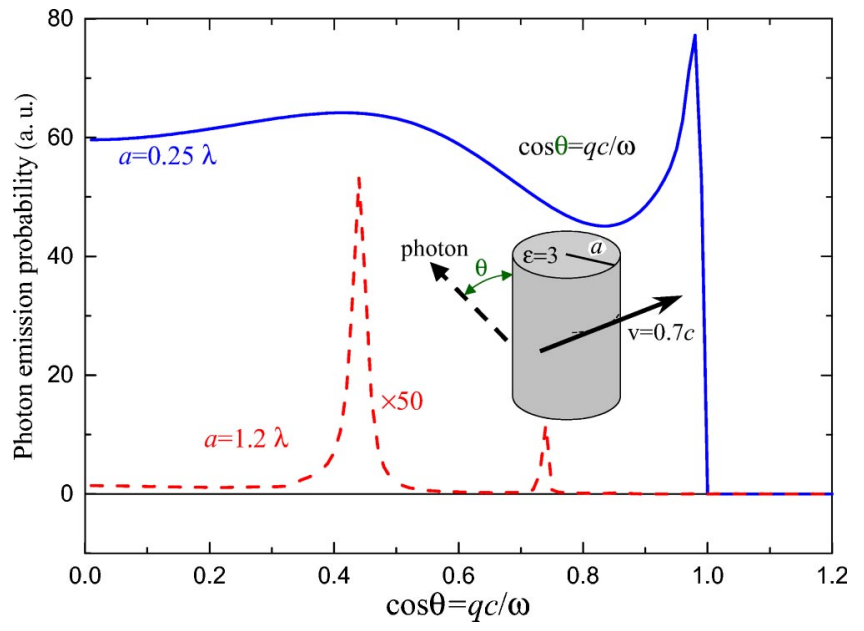

FIG. 5. (Color online) Angular distribution of the radiation induced by a unit point charge following a grazing trajectory perpendicular to a cylinder described by $\epsilon=3$. The emission intensity has been integrated over azimuthal directions of emission with respect to the cylinder axis. The velocity $v$ and cylinder radius $a$ are given in the inset and labels, and so is the relation between the emission angle $\theta$ and the momentum transfer parallel to the cylinder, $q$. The dashed curve, corresponding to $a=1.2 \lambda$, has been divided by a factor of 50 for the sake of readability. The probability is given in atomic units for a photon energy of $3 \mathrm{eV}$.

where $\gamma_{j}=1 / \sqrt{1-k_{j}^{2} / q^{2}}$ (using the notation of Sec. II), $\mathbf{E}_{j}^{\text {bulk }}$ is given by Eq. (3), $\mathbf{R}$ are the coordinates perpendicular to the $z$ axis, and the integral over $l$ is extended along points $\mathbf{R}_{l}$ of the boundary contours of medium $j$. The BEM provides the numerical solution of the boundary currents and charges, $\mathbf{h}_{j}(l, q)$ and $\sigma_{j}(l, q)$, in the presence of a given external perturbation (the moving charge in our case) for each value of $q$ on a set of representative points along the boundary contours.

For dielectric objects of real dielectric function, all energy losses experienced by the moving charge must be converted into radiation emission, since the medium is unable to dissipate any of this energy. Therefore, the radiation emission can be calculated from the energy loss, and this is what has been done in this section by inserting Eq. (19) into Eq. (9).

In particular, the loss probability can be separated into the contribution of each $q$, that is, into the contribution of radiation emission with momentum $q$ along the $z$ axis. Thus, for a translationally invariant object surrounded by vacuum, this radiation will be emitted forming an angle $\theta$ with the $z$ axis such that $\cos \theta=q /(\omega / c)$. This is the case considered in Fig. 5 for grazing incidence on a cylinder surface, where the emission probability is represented as a function of emission angle. Note that no losses occur for $q>\omega / c$, which would $\mathrm{be}$, in principle, possible if guided modes of the cylinder (confined by total internal reflection) where excited for $\omega / c$ $<q<\omega / c \sqrt{\epsilon}$ (with $\epsilon=3$ ), but no such modes exist for the radii under consideration. For $a=1.2 \lambda$ (broken curve) two radiative quasiguided modes are excited in the cylinder, which show up as peaks in the angular distribution of emitted photons. These peaks have certain broadening in $q$, indicating that they correspond to excitation of modes that have

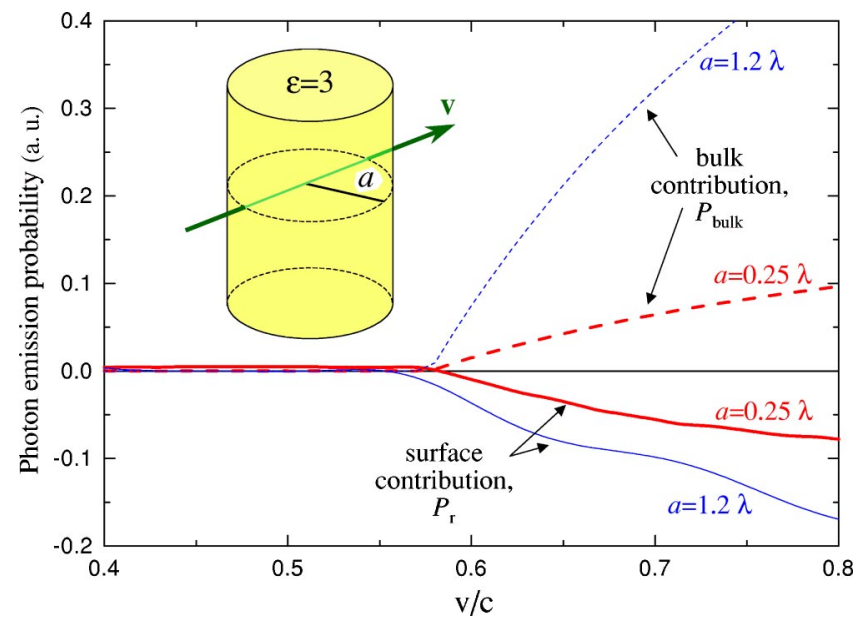

FIG. 6. (Color online) Velocity dependence of the bulk and surface contribution to the radiation emission probability for a unit point charge passing by the center of a cylinder and moving perpendicular to its axis. Two different values of the cylinder radius have been considered, as shown by labels, expressed in terms of the wavelength. The probability is given in atomic units for a photon energy of $3 \mathrm{eV}$.

a finite lifetime, which can be in turn attributed to coupling to freely propagating radiation, so that they constitute whispering gallery modes that are actually leaking out from the cylinder. For much thinner cylinders $(a=0.25 \lambda)$, no guided mode is possible, as it should be in the electrostatic limit which is valid for $a \ll \lambda$, so that the resulting emission is strongly attenuated and originates in the polarization of the surface without coupling to any particular resonance of the medium.

A similar analysis has been carried out for penetrating trajectories in Fig. 6, where the emission probability is given as a function of velocity for trajectories intersecting the axis of cylinders of different thickness. In this case, the trajectory evolves partly in vacuum and partly inside the material, so that care must be taken when applying Eq. (9) over regions of different $j$ [see Eq. (19)]. The result has been separated into the contribution of bulk and surface loss probabilities. The former is defined as the probability obtained from Eq. (2) multiplied by the diameter of the cylinder, that is, the probability that would be obtained if the emission came only from the part of the trajectory that lies inside the medium and if the loss probability per unit time in the medium was given by the expression obtained for an infinite homogeneous medium; this is a natural separation because the bulk loss contribution comes exclusively from the first term in Eq. (19). The surface loss is taken as the total loss probability minus the bulk loss probability, as obtained from the boundary integral in Eq. (19). For a cylinder much thinner than the wavelength (radius $a=0.25 \lambda$ ) these two contributions almost cancel each other, so that no significant emission is produced, again in agreement with the expected result in the electrostatic limit. Actually, the fact that the contribution of the surface is negative can be understood as another aspect of the so-called begrenzung effect, first described in films by Ritchie, ${ }^{42}$ and consisting in that the excitation of surface 


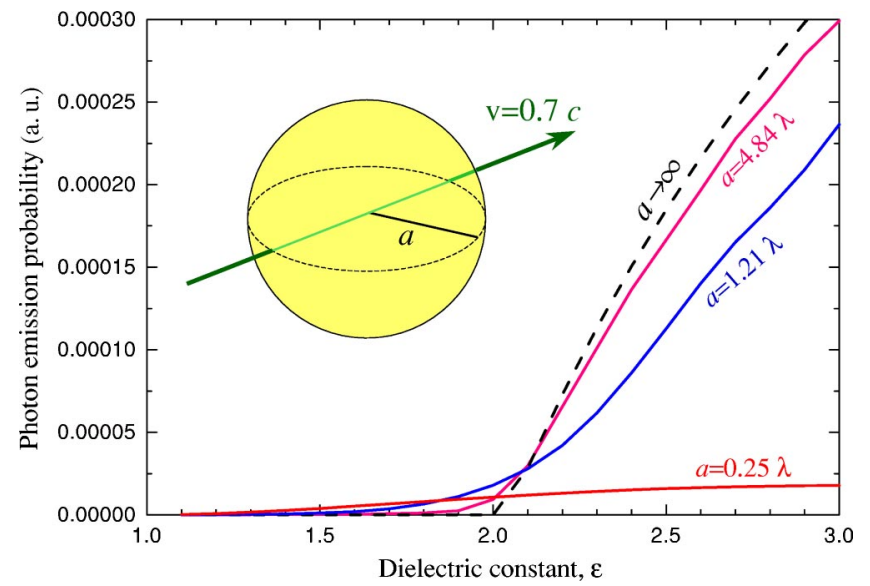

FIG. 7. (Color online) Dependence of the radiation emission probability for a unit point charge passing by the center of a sphere on its dielectric function $\epsilon$. Various values of the sphere radius have been considered, as shown by labels. The emission probability has been divided by the diameter of the sphere in each case, and it is given in atomic units for a photon energy of $3 \mathrm{eV}$.

modes is made at the expense of bulk modes. However, the bulk contribution (linear with the diameter) is dominant for thicker cylinders $(a=1.2 \lambda)$, and one would expect that the surface contribution converges actually to a finite value as the diameter of the cylinder increases: this would be essentially associated to transition radiation, similar to the perpendicular trajectory in the thin film discussed in Sec. II C.

\section{B. Spheres}

The convergence towards the bulk limit (2) when a charge crosses a finite object of increasing size is illustrated in Fig. 7 for dielectric spheres. These results have been obtained using the BEM for axially symmetric samples, for which the electric field can be separated into different azimuthal components $m$, resulting in an equation similar to Eq. (19) but with a more involved expression for the Green function. Starting from the results of Ref. 29, the electric field in the far field limit can be written

$$
\mathbf{E}(\mathbf{r})=\mathbf{E}_{j}^{\mathrm{bulk}}(\mathbf{r})+\frac{e^{\mathrm{i} k_{j} r}}{r} \mathbf{f}(\hat{\mathbf{r}}),
$$

where

$$
\begin{aligned}
\mathbf{f}(\hat{\mathbf{r}})= & \sum_{m} \mathrm{i}^{1-m} \int d l \rho_{l}\left[\frac{\omega}{c} \mathbf{h}_{j}^{m}(l)-k_{j} \sigma_{j}^{m}(l) \hat{\mathbf{r}}\right] \\
& \times J_{m}\left(k_{j} \rho_{l} \sin \theta\right) \mathrm{e}^{-\mathrm{i} k_{j} \cos \theta z} l \mathrm{e}^{\mathrm{i} m \phi},
\end{aligned}
$$

is the boundary contribution far-field amplitude, $\left(\rho_{l}, 0, z_{l}\right)$ are cylindrical coordinates of the boundary points along which the $l$ integral is performed, and $(r, \theta, \phi)$ are the spherical coordinates of $\mathbf{r}$. The boundary currents and charges $\mathbf{h}_{j}^{m}(l)$ and $\sigma_{j}^{m}(l)$ have been obtained numerically using the BEM. ${ }^{29}$ Then, the boundary contribution (i.e., disregarding $\mathbf{E}_{j}^{\text {bulk }}$ ) to the angle-resolved far-field emission probability is easily expressed in terms of $\mathbf{f}(\hat{\mathbf{r}})$ as $^{26}\left(\epsilon_{j} / 4 \pi^{2} k_{j}\right)|\mathbf{f}(\hat{\mathbf{r}})|^{2}$, from which, upon integration over emission directions, one obtains the total emission probability. This is the procedure that we have followed to obtain Fig. 7, rather than calculating the energy loss probability. Furthermore, the sphere under consideration is surrounded by vacuum, and consequently, $\mathbf{E}_{j}^{\text {bulk }}$ is evanescent and does not contribute to the far field.

Figure 7 shows how the photon emission probability calculated by means of the BEM and normalized to the diameter (i.e., the length of the trajectory inside the sphere) converges rapidly to the bulk limit ( $a \rightarrow \infty$, broken curve) as the radius exceeds the wavelength $\lambda$. Like in the case of small cylinders, small spheres give rise to a relatively poor emission. It is important to recall that all losses are converted into photon emission in this case, since the sphere has real dielectric function that makes it unable to absorb energy out of the moving charge. Coupling to whispering gallery modes is actually part of this emission scheme: these modes have a finite radiative lifetime ${ }^{43}$ so that they give rise to photon emission eventually after they are excited (i.e., no trapped modes exist in a dielectric sphere, since all of them have eigenfrequencies with nonzero imaginary parts ${ }^{43}$ that represent a rate of leakage into freely propagating radiation). Therefore, the entire Cherenkov emission emerges eventually from the sphere, and the fact that the bulk limit is recovered for large spheres seems to indicate that there are no significant interference effects between the direct bulk emission and internally reflected components, just as it occurred in the case of the semi-infinite medium of Sec. II. This equivalence between total emission probability and energy loss probability for materials with real dielectric constant is further tested below and compared to experimental results of EELS in dielectric spheres.

Radiation can also be produced when external charges are passing near dielectric spheres, as shown in Fig. 8 for 200 $\mathrm{keV}$ electrons and $\mathrm{Al}_{2} \mathrm{O}_{3}$ spheres. In this case, we have adopted a previously obtained analytical expression for the energy loss probability that includes retardation, ${ }^{41,44}$

$$
\begin{aligned}
P(\omega)= & \frac{1}{c \omega} \sum_{l=1}^{\infty} \sum_{m=-l}^{l} \frac{1}{l(l+1)} K_{m}^{2}\left(\frac{\omega b}{v \gamma}\right) \\
& \times\left[\left|\frac{2 m v}{c} A_{l m}\right|^{2} \operatorname{Im}\left\{t_{l}^{M}\right\}+\left|\frac{1}{\gamma} B_{l m}\right|^{2} \operatorname{Im}\left\{t_{l}^{E}\right\}\right],
\end{aligned}
$$

where $\gamma=1 / \sqrt{1-v^{2} / c^{2}}$ accounts for the contraction of the frequency $\omega$ as seen by the moving charge, the scattering matrix elements $t_{l}^{M}$ and $t_{l}^{E}$ depend exclusively on the sphere composition and radius (their analytical expressions can be found in Ref. 44), $b$ is the impact parameter with respect to the center of the sphere, and the dimensionless coefficients $A_{l m}$ and $B_{l m}$ depend only on the ratio $\beta=v / c$ as

$$
\begin{aligned}
A_{l m}= & \sqrt{\frac{2 l+1}{\pi} \frac{(l-|m|) !}{(l+|m|) !}} \\
& \times(2|m|-1) ! ! \frac{\mathrm{i}^{l+|m|} s_{m}}{\beta(\beta \gamma)^{|m|}} C_{l-|m|}^{(|m|+1 / 2)}\left(\frac{1}{\beta}\right),
\end{aligned}
$$

and 


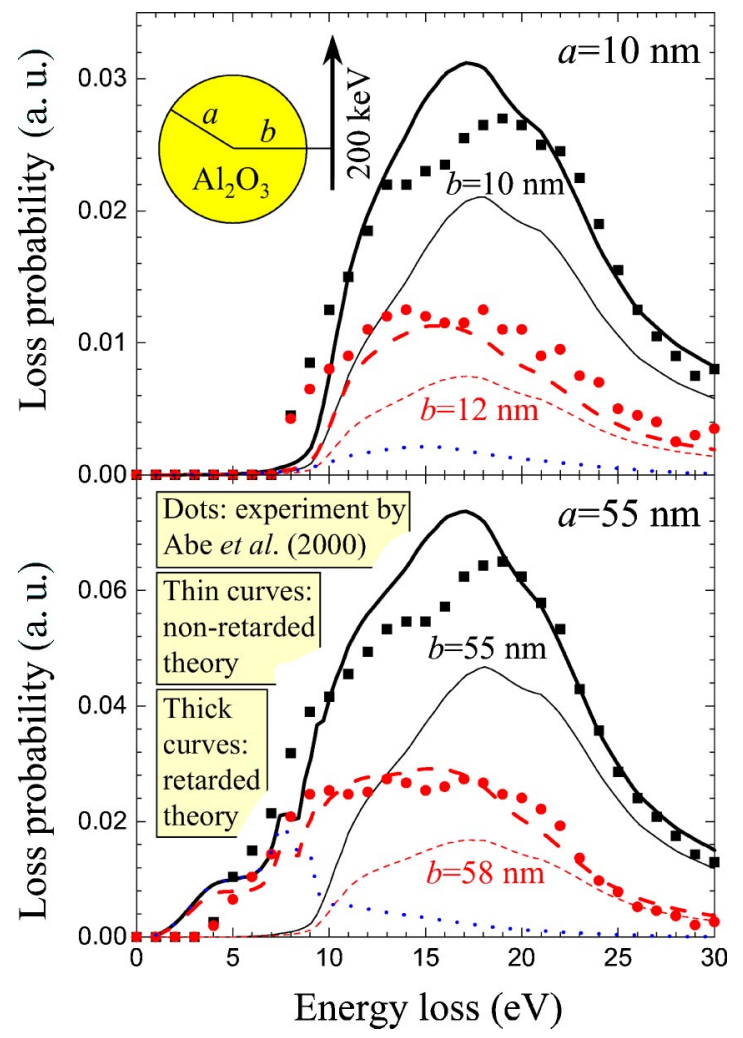

FIG. 8. (Color online) Loss probability for electrons passing near aluminum oxide spheres of radius $a=10 \mathrm{~nm}$ (upper plot) and $a=55 \mathrm{~nm}$ (lower plot). The experimental data taken from Ref. 46 are compared here with retarded (thick curves) and non-retarded (thin curves) calculations for the energy loss. The impact parameter with respect to the sphere surface is $b-a=0$ in the continuous curves, $b-a=2 \mathrm{~nm}$ in the broken curves of the upper figure, and $b-a=3 \mathrm{~nm}$ in the broken curves of the lower figure. The calculated probability is given in atomic units, whereas the experimental results have been multiplied by a single constant value for both curves in the upper plot, and by another single constant value for both curves in the lower plot.

$$
\begin{aligned}
B_{l m}= & A_{l m+1} \sqrt{(l+m+1)(l-m)} \\
& -A_{l m-1} \sqrt{(l-m+1)(l+m)} .
\end{aligned}
$$

Here, $s_{m}=1$ if $m \geqslant 0, s_{m}=(-1)^{m}$ if $m<0, K_{m}$ is the modified Bessel function of order $m$, and $C_{m}^{(\nu)}$ is the Gegenbauer polynomial. $^{45}$

The experimental data of Abe et al. ${ }^{46}$ (points in Fig. 8) are well reproduced by the retarded calculation of Eq. (22) (thick curves) using optical data to model the dielectric function of $\mathrm{Al}_{2} \mathrm{O}_{3} \cdot{ }^{36}$ In particular, substantial energy losses take place below $7 \mathrm{eV}$ for large spheres (lower figure, $55 \mathrm{~nm}$ radius), where the dielectric function of alumina is real, ${ }^{36}$ so that energy absorption by the medium is negligible. Therefore, these low-energy losses must originate in the emission of radiation induced by the passage of the electrons, and this constitutes a genuine manifestation of retardation effects that cannot be explained by the nonretarded counterpart of Eq. (22) (thin curves), which was first obtained by Ferrell and
Echenique $^{47,46}$ [their expression is fully recovered from Eq. (22) in the $c \rightarrow \infty$ limit] and which does not account for radiation effects.

This hypothesis has been further tested by calculating the radiation emission probability for the grazing trajectories under consideration (dotted curves), which equals the loss probability below $7 \mathrm{eV}$, but is well below it above $10 \mathrm{eV}$, where most of the losses go into energy dissipation in the particle. The emission radiation probability has been calculated from an analytical expression derived in Refs. 41 and 44. That expression is still given by Eq. (22) if one substitutes $\operatorname{Im}\left\{t_{l}^{\nu}\right\}$ by $\left|t_{l}^{\nu}\right|^{2}$, with $\nu=M, E$. In fact, one always has $\operatorname{Im}\left\{t_{l}^{\nu}\right\} \geqslant\left|t_{l}^{\nu}\right|^{2}$, where the equal applies precisely for real dielectric functions, ${ }^{44}$ as in our case below $7 \mathrm{eV}$.

The radiation produced in these experiments, where aloof electron beams are used that do not touch the material, originates in the induced charges and currents produced by the passage of the electrons. Indeed, these induced sources evolve in time in a bounded object (the $\mathrm{Al}_{2} \mathrm{O}_{3}$ spheres), so that they are necessarily subject to acceleration, and therefore, they radiate. In brief, the emission is mediated by multipole whispering gallery modes excited by the passing electrons in the spheres over a wide range of frequencies, which ultimately leak out as free radiation.

\section{Buried structures}

We have finally analyzed the angular dependence of the Cherenkov emission when a point charge passes near a buried structure. Just as in the case of a penetrating trajectory in a dielectric sphere, this has been done numerically by using the BEM for axially symmetric geometries, ${ }^{29}$ as explained in Sec. III B [see Eq. (21)]. Figure 9 shows angular patterns of emission for buried axially symmetric ellipsoidal voids of different aspect ratios for various impact parameters of the probe trajectory, $b$. The bulk Cherenkov cone coming from $\mathbf{E}_{j}^{\text {bulk }}$ in Eq. (20) has been represented just by dashed curves in Fig. 9, since this contribution is infinitesimally peaked at the Cherenkov cone angle.

In general, the scattered light (dark regions) piles up near the intersection of the Cherenkov cone with the plane defined by the trajectory and the void center. In particular, the large impact-parameter limit $(b \rightarrow \infty)$ corresponds to the case where a radiation plane wave is scattered by the void from an incoming direction dictated by the Cherenkov angle. However, for small impact parameters the angular patterns show a rich structure that varies strongly with the shape of the object, suggesting the possibility of employing this effect to analyze buried structures in transparent materials, combining in this way the nanometer spatial resolution of electron microscopes and the possibility of measuring different wavelengths (i.e., changing the size of the object relative to the wavelength) with the excellent energy resolution of light detectors.

Interestingly, convergence towards the large $b$ limit is rather slow: near-field and curve wave-front effects in the Cherenkov emission are still making a dramatic contribution at a separation of $b=11 \lambda$, and this is most clear for elongated structures (lower part of Fig. 9). The absolute maxi- 


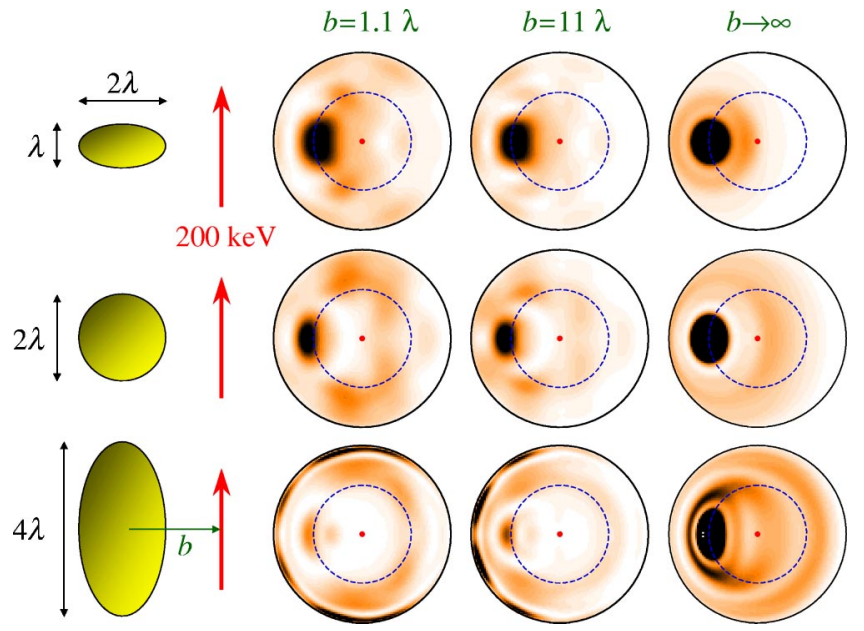

FIG. 9. (Color online) Angular dependence of the CR emission for a $200 \mathrm{keV}$ electron moving in a medium described by $\epsilon=3$ and passing near ellipsoidal voids, as shown in the insets. The trajectory is parallel to the axis of symmetry of the voids. Different aspect ratios and impact parameters $b$ are considered, as shown by labels. Darker regions correspond to higher intensity. The plots represent the angular emission in a stereographic projection of the upper hemisphere, as defined by the trajectory, represented by a point at the center of each contour plot. The bulk Cherenkov cone emission lies along the dashed circles (this part of the emission is independent of the azimuthal direction and proportional to the overall trajectory length, so that it has not been represented in the plots). Distances are given in terms of the radiation wavelength $\lambda$.

mum intensity of emission outside the Cherenkov cone decreases smoothly with $b$ as $1 / b$, which permits in principle sampling trajectories passing relatively far away from the buried objects. This $1 / b$ behavior can be easily understood in the case of the sphere, for which the emission probability excluding the Cherenkov cone is given analytically by an expression similar to Eq. (22), where $\gamma$ has to be replaced by $1 / \sqrt{1-\epsilon v^{2} / c^{2}}$, which becomes imaginary under the Cherenkov condition, so that the Bessel function $K_{m}$ has an imaginary argument and oscillates with $b$ while its modulus decays like $1 / \sqrt{b}$.

\section{SUMMARY}

We have shown that the CR emission is strongly modified by the presence of dielectric boundaries. The case of a planar surface shows that $\mathrm{CR}$ can be produced inside the medium by the induced charges accompanying the external charge, even when this is outside the material (see Fig. 1). For motion inside the medium, the emission probability oscillates with the distance to the surface, as a signature of interference effects between direct Cherenkov emission and its total reflection at the inner dielectric boundary.

In thin dielectric films, only guided modes can be excited, which in the case of trajectories parallel to the film surfaces implies that the losses are restricted to specific values of momentum and energy transfer, as defined by the dispersion relation of those modes. Electrons crossing thin films are capable of exciting guided modes as well, and the theory presented above describes reasonably well available experiments. $^{35}$

When a dielectric is confined along two spatial directions, such as in cylinders, the emission induced by an external charge moving along the translational direction of symmetry can only take place for discretized wavelengths that resonate with the propagating modes of the cylindrical wave guide. This confined modes have been also shown to couple to point charges moving perpendicularly to dielectric cylinders, giving rise to resonant features in the transition radiation that they produce.

The bulk Cherenkov limit has been recovered for charges crossing spheres of increasingly large radius. In the opposite limit of small radius, the emission from both spheres and cylinders is strongly suppressed, since losses in this regime can be well described within the electrostatic limit, where no radiation is allowed.

Finally, the angular distribution of Cherenkov emission has been analyzed for electrons passing near voids in dielectrics. A strong dependence on impact parameter and void shape is obtained, suggesting the possibility of using this technique to provide some sort of holographic reconstruction of buried structures.

\section{ACKNOWLEDGMENTS}

The authors want to thank Professor A. Howie for many stimulating conversations and helpful suggestions. Help and support from the Basque Departamento de Educación, Universidades e Investigación, the University of the Basque Country UPV/EHU (Contract No. 00206.215-13639/2001), and the Spanish Ministerio de Ciencia y Tecnología (Contract No. MAT2001-0946) is gratefully acknowledged.

\section{APPENDIX: THE ANISOTROPIC FILM UNDER OBLIQUE INCIDENCE}

The formalism presented in Sec. II for planar interfaces can be easily generalized to deal with anisotropic media and oblique incidence. Solving Maxwell's equations in momentum space, one can generalize the plane waves of Sec. II to the expressions

$$
\mathbf{K}_{\mathbf{Q}}^{ \pm}=\left(\mathbf{Q}, \pm k_{z j}^{\sigma}\right)
$$

for the momentum and

$$
\hat{\mathbf{e}}_{\mathbf{Q}, p}^{j \pm}=\frac{C}{k Q}\left( \pm \mathbf{Q} k_{z j}^{p} / \epsilon_{j}^{\|},-Q^{2} / \epsilon_{j}^{\perp}\right)
$$

for the polarization vector of $p$ waves [the polarization vector of $s$ waves is still given by Eq. (4)]. Here,

$$
k_{z j}^{s}=\sqrt{k^{2} \epsilon_{j}^{\|}-Q^{2}}, \quad k_{z j}^{p}=\sqrt{k^{2} \epsilon_{j}^{\|}-Q^{2} \epsilon_{j}^{\|} / \epsilon_{j}^{\perp}},
$$

$\epsilon_{j}^{\|}$and $\epsilon_{j}^{\perp}$ denote the dielectric function of medium $j$ for polarization along the directions parallel and perpendicular to the film surfaces, respectively, and the constant $C$ is chosen such that $\hat{\mathbf{e}}_{\mathbf{Q}, p}^{j \pm}$ has unit norm.

The bulk field for oblique incidence takes a relatively simple form when the parallel momentum $\mathbf{Q}$ is contained in 
the plane determined by the surface normal and the velocity vector. In particular, choosing the latter within the $x-z$ plane $\left[\mathbf{v}=\left(v_{x}, 0, v_{z}\right)\right]$, one obtains $\left(Q_{y}=0\right)$

$$
\begin{gathered}
E_{j x}^{\text {bulk }}\left(Q_{x}, 0, z\right)=\left[\left(k^{2} \epsilon_{j}^{\perp}-Q_{x}^{2}\right) v_{x} / v_{z}-Q_{x} q_{z}\right] F, \\
E_{j z}^{\text {bulk }}\left(Q_{x}, 0, z\right)=\left[\left(k^{2} \epsilon_{j}^{\|}-q_{z}^{2}\right)-Q_{x} q_{z} v_{x} / v_{z}\right] F, \\
H_{j y}^{\text {bulk }}\left(Q_{x}, 0, z\right)=\left(q_{z} E_{j x}-Q_{x} E_{j z}\right) / k
\end{gathered}
$$

*Email address: jga@sw.ehu.es

${ }^{1}$ J.D. Jackson, Classical Electrodynamics (Wiley, New York, 1975).

${ }^{2}$ P.A. Cherenkov, Dokl. Akad. Nauk SSSR 2, 451 (1934).

${ }^{3}$ I.M. Frank and I. Tamm, Dokl. Akad. Nauk SSSR 14, 109 (1937).

${ }^{4}$ I. Tamm, J. Phys. (Moscow) 1, 439 (1939); E. Fermi, Phys. Rev. 57, 485 (1940).

${ }^{5}$ E. Lalor and E. Wolf, Phys. Rev. Lett. 26, 1274 (1971).

${ }^{6}$ D.N. Patro, Phys. Rev. Lett. 49, 1083 (1982).

${ }^{7}$ J.V. Julley, Cherenkov Radiation and its Application (Pergamon, New York, 1958).

${ }^{8}$ See, for example, P.W. Gorham, D.P. Saltzberg, P. Schoessow, W. Gai, J.G. Power, R. Konecny, and M.E. Conde, Phys. Rev. E 62, 8590 (2000), for an application of Cherenkov radiation to the detection of high-energy neutrinos.

${ }^{9}$ V.P. Zrelov and J. Ruzicka, Czech. J. Phys., Sect. B 39, 368 (1989); J. Ruzicka and V.P. Zrelov, Czech. J. Phys. 43, 551 (1993); M. Ciljak et al., Nucl. Instrum. Methods Phys. Res. A 498, 126 (2003).

${ }^{10}$ B.M. Bolotovskii and A.V. Serov, Nucl. Instrum. Methods Phys. Res. B 201, 49 (2003); E.D. Gazazian, K.A. Ispirian, and M.I. Ivanian, ibid. 201, 84 (2003).

${ }^{11}$ E. Goldobin, A. Wallraff, N. Thyssen, and A.V. Ustinov, Phys. Rev. Lett. 57, 130 (1998).

${ }^{12}$ I. Carusotto, M. Artoni, G.C. La Rocca, and F. Bassani, Phys. Rev. Lett. 87, 064801 (2001).

${ }^{13}$ R.M. More, Phys. Rev. Lett. 16, 781 (1966).

${ }^{14}$ W.D. Kimura, G.H. Kim, R.D. Romea, L.C. Steinhauer, I.V. Pogorelsky, K.P. Kusche, R.C. Fernow, and X. Wang, Phys. Rev. Lett. 74, 546 (1995).

${ }^{15}$ T.E. Stevens, J.K. Wahlstrand, J. Kuhl, and R. Merlin, Science 291, 627 (2001).

${ }^{16}$ D. Schieber and L. Schächter, Phys. Rev. E 57, 6008 (1998).

${ }^{17}$ E. Kröger, Z. Phys. 216, 115 (1968).

${ }^{18}$ A.A. Lucas and E. Kartheuser, Phys. Rev. B 1, 3588 (1970).

${ }^{19}$ I.N. Onishchenko, D.Yu. Sidorenko, and G.V. Sotnikov, Phys. Rev. E 65, 066501 (2002).

${ }^{20}$ N. Akhmediev and M. Karlsson, Phys. Rev. A 51, 2602 (1995).

${ }^{21}$ D.V. Skryabin, F. Luan, J.C. Knight, and P.St.J. Russell, Science 301, 1705 (2003).

${ }^{22}$ B. Lastdrager, A. Tip, and J. Verhoeven, Phys. Rev. E 61, 5767 (2000).

${ }^{23}$ J.P.R. Bolton and M. Chen, J. Phys.: Condens. Matter 7, 3373 (1995); 7, 3389 (1995); 7, 3405 (1995). (the rest of the components are zero), where

$$
F=\frac{(4 \pi \mathrm{i} k / c) e^{\mathrm{i} q_{z} z}}{\left(Q_{x} q_{z}\right)^{2}-\left(k^{2} \epsilon_{j}^{\perp}-Q_{x}^{2}\right)\left(k^{2} \epsilon_{j}^{\|}-q_{z}^{2}\right)}
$$

and $q_{z}=\left(\omega-Q_{x} v_{x}\right) / v_{z}$.

${ }^{24}$ C. Luo, M. Ibanescu, S.G. Johnson, and J.D. Joannopoulos, Science 299, 368 (2003).

${ }^{25}$ F.J. García de Abajo, A.G. Pattantyus-Abraham, N. Zabala, A. Rivacoba, M.O. Wolf, and P.M. Echenique, Phys. Rev. Lett. 91, 143902 (2003).

${ }^{26}$ F.J. García de Abajo, Phys. Rev. Lett. 82, 2776 (1999); Phys. Rev. B 60, 6086 (1999).

${ }^{27}$ S. Yamaguti, J. Inoue, O. Haeberlé, and K. Ohtaka, Phys. Rev. B 66, 195202 (2002).

${ }^{28}$ F.J. García de Abajo and L.A. Blanco, Phys. Rev. B 67, 125108 (2003).

${ }^{29}$ F.J. García de Abajo and A. Howie, Phys. Rev. B 65, 115418 (2002).

${ }^{30}$ R. García-Molina, A. Gras-Martí, A. Howie, and R.H. Ritchie, J. Phys. C 18, 5335 (1985).

${ }^{31}$ P. Moreau, N. Brun, C.A. Walsh, C. Colliex, and A. Howie, Phys. Rev. B 56, 6774 (1997).

${ }^{32}$ Transition radiation was predicted by V.L. Ginzburg and I.M. Frank, Sov. Phys. JETP 16, 15 (1946). See N. Yamamoto, H. Sugiyama, and A. Toda, Proc. R. Soc. London, Ser. A 452, 2279 (1996), and references therein for related experiments.

${ }^{33}$ M.L. Ter-Mikaelian, High-Energy Electromagnetic Processes in Condensed Media (Wiley, New York, 1972).

${ }^{34}$ R. Vincent and J. Silcox, Phys. Rev. Lett. 31, 1487 (1973).

${ }^{35}$ C.H. Chen and J. Silcox, Phys. Rev. Lett. 35, 390 (1975).

${ }^{36}$ E.D. Palik, Handbook of Optical Constants of Solids (Academic Press, New York, 1985).

${ }^{37}$ A. Otto and R.B. Swan, Z. Phys. 206, 277 (1967).

${ }^{38}$ R.H. Ritchie and H.B. Eldridge, Phys. Rev. 126, 1935 (1962).

${ }^{39}$ N. Zabala, A. Rivacoba, and P.M. Echenique, Surf. Sci. 209, 465 (1989).

${ }^{40}$ H.A. Olsen and H. Kolbenstvedt, Phys. Rev. A 21, 1987 (1980).

${ }^{41}$ F.J. García de Abajo and A. Howie, Phys. Rev. Lett. 80, 5180 (1998).

${ }^{42}$ R.H. Ritchie, Phys. Rev. 106, 874 (1957).

${ }^{43}$ R. Fuchs and K.L. Kliewer, J. Opt. Soc. Am. 58, 319 (1968).

${ }^{44}$ F.J. García de Abajo, Phys. Rev. B 59, 3095 (1999).

${ }^{45}$ M. Abramowitz and I.A. Stegun, Handbook of Mathematical Functions (Dover, New York, 1972).

${ }^{46}$ H. Abe, H. Kurata, and K. Hojou, J. Phys. Soc. Jpn. 69, 1553 (2000).

${ }^{47}$ T.L. Ferrell and P.M. Echenique, Phys. Rev. Lett. 55, 1526 (1985). 